\title{
Caracterización morfológica y molecular de una colección de aislamientos de Phomopsis longicolla (teleomorfo desconocido: Diaporthales) de la región templada y subtropical de Argentina
}

\author{
Facundo E. Hernández ${ }^{1}$, Rosanna N. Pioli ${ }^{*}$, Alejandra M. Peruzzo ${ }^{1}$, Ángela N. Formento ${ }^{2}$ \&
} Guillermo R. Pratta ${ }^{3}$

1. Fitopatología y Botánica Criptogámica, Laboratorio de Biodiversidad Vegetal y Microbiana (BioVyM). Facultad de Ciencias Agrarias (FCA), Universidad Nacional de Rosario (UNR). Campo Experimental J. Villarino, CC14. (S2125ZA) Zavalla, Santa Fe. Argentina; hernandezfacundo@live.com, rpioli@unr.edu.ar, rosanna.pioli@gmail.com

2. Área Sanidad y Protección Vegetal, Estación Experimental INTA Paraná, Oro Verde, Entre Ríos; formento.angela@inta.gob.ar

3. Genética y Laboratorio PRAMIN, FCA, UNR. Campo Experimental J. Villarino, CC14. (S2125ZA) Zavalla, Santa Fe. Argentina; gpratta@unr.edu.ar

* Correspondencia

Recibido 16-IX-2014. Corregido 25-II-2015. Aceptado 26-III-2015.

\begin{abstract}
Morphologic and molecular characterization of Phomopsis longicolla (teleomorph unknown: Diaporthales) from tempered and subtropical regions of Argentina. Diaporthe (teleomorpho)-Phomopsis (anamorph) (DP) is a fungal group of great genetic diversity with over 900 species associated to a wide host range that includes cultivated and uncultivated species, forest, fruit trees and weeds. DP isolates are hemibiotrophs and have different sources of primary inoculum as stubble and seeds to restart cycles of parasitism saprophytism. They colonize host tissues from early plant stages and establish different nutritional relationships, acting as endophytic and necrotrophic fungi. The plasticity of the Phomopsis genus has favored its expansion to different agro-ecosystems and various hosts constituting an epidemiological risk. The objective was to validate the identity and evaluate the biological relationships among 12 isolates of $P$. longicolla and D. phaseolorum var. sojae (anamorph P. phaseoli var. sojae) obtained in different tempered and subtropical agro-environments of Argentina, in order to analyze the variability and strategies for preserving fungal biodiversity. Macromorphological attributes (such as texture and color of colonies, stroma shape and distribution, pycnidia and perythecia shape and distribution) and micro-morphological characteristics (such as size and shape of conidia, asci and ascospores) allowed identifying three new isolates as P. longicolla. A complementary molecular analysis was also made to overcome the limitations derived from the morphological analysis, thus the AFP.8413 isolate was finally identified as $P$. longicolla. The molecular characterization was useful to identify the evaluated isolates and to group them in four taxa of the Diaporthe-Phomopsis complex: ten isolates were included in $P$. longicolla, one isolate was included in D. phaseolorum var. sojae (anamorph $P$. phaseoli var. sojae), one isolate was identified as D. phaseolorum var. caulivora and two isolates were included in D. phaseolorum var. meridionalis. The use of phenotipic and molecular tools have contributed to an accurate identification of P. longicolla, and comprehension about the biological relationships (homo or heterothallic hibridizations) among D. phaseolorum varieties (P. phaseoli) and species of Diaporthe-Phomopsis. This allowed also a better understanding of the mechanisms of fungic plasticity, to colonize and expand their host range and genetic variability, promoting thus their biodiversity conservation. Rev. Biol. Trop. 63 (3): 871-884. Epub 2015 September 01.
\end{abstract}

Key words: hemi-biotrophic fungus, Phomopsis longicolla, epidemiology, cluster analysis, agro-biology.

Diaporthe-Phomopsis $(D P)$ constituye un grupo de amplia diversidad genética con más de 900 especies distribuidas en un amplio rango de hospedantes que incluye especies cultivadas y no cultivadas, forestales nativas, frutales y malezas (Rossman, Farr, \& Castlebury, 2007). Los aislamientos de $D P$ colonizan los tejidos del hospedante de manera temprana 
y establecen diferentes relaciones nutricionales tales como endofitia, necrotrofia, saprofitismo y parasitismo (Kleczewski, Bauer, Bever, Clay, \& Reynolds, 2012). Se reconocen como endófitos aquellos hongos que desarrollan al menos una etapa de su vida asociados a tejidos vegetales sin causar síntomas patogénicos en la planta hospedante, la cual simplemente provee un nicho disponible y apto para el desarrollo fúngico (Crous \& Groenewald, 2005; Udayanga et al., 2011). Algunos Phomopsis endófitos fueron aislados de Magnolia liliflora (Familia Magnoliaceae), Panicum virgatum (Familia Poaceae), Vaccinium corymbosum y $V$. macrocarpon (arándanos) (F. Ericaceae), Prunus sp. (F. Rosaceae) y Carthamus lanatus (F. Asteraceae) entre otros (Farr, Castlebury, \& Rossman, 2002; Promputtha, Jeewon, Lumyong, McKenzie, \& Hyde, 2005; Ash et al., 2010; Linnakoski, Puhakka-Tarvainen, \& Pappinen, 2012). Sin embargo, la definición de endófitos incluye, además, a aquellas especies patógenas que desarrollan una fase o período de latencia prolongada durante su ciclo de vida (Saikkonen, 2007; Sakadilis, Hardy, \& Burgess, 2011). En ese contexto, se han descrito numerosos géneros fúngicos necrotróficos $\mathrm{o}$ hemi-biótrofos, entre ellos Phomopsis, con una fase endofítica y/o parasitaria asociadas a la planta y una fase saprofítica asociada a los residuos orgánicos (Heller \& Gierth, 2001; Debaeke \& Moinard, 2010).

Entre las especies de Phomopsis ( $P$. phaseoli, $P$. longicolla y $P$. helianthi) presentes en Argentina, $P$. longicolla es una de las más expandidas y relevantes por estar asociadas a Glycine y otros hospedantes (Pioli \& Morandi, 2005). G. $\max$ (L. Merr.) y su progenitor silvestre (G. soja) pertenecen a la familia Fabaceae (Sub tribu Phaseolaeae) y se originaron a partir de seis sitios geográficamente distribuidos en Japón, Korea, Rusia y tres regiones de China (Ying-Hui et al., 2010). La especie cultivada se siembra extensivamente a nivel mundial por ser una fuente primaria de proteínas, aceites, productos derivados y bio-combustibles. Incluye además variedades productoras de semillas y granos cosechados maduros y secos, y variedades hortícolas para consumo de brotes, granos frescos o verdes (soja edamame) (Ponce de León, Torija, \& Matallana, 2013). Las interacciones patogénicas de G. max con $P$. longicolla (Plo) Hobbs y P. phaseoli var. sojae (Pps) (teleomorfo D. phaseolorum var. sojae) producen el Tizón del tallo y vainas y luego el Decaimiento o podredumbre de semillas en soja (TTVyS), afectan significativamente el número, el peso y la calidad de semillas y granos secos, como también los frutos y granos para consumo verde (Pioli, Benavídez, \& Morandi, 1997; Pioli, Benavídez, Morandi, \& Bodrero, 2000; Meriles, Lamarque, Labuckas, \& Maestri, 2004; Benavídez, Pioli, \& Morandi, 2010). Hobbs, Schmitthenner y Kuter (1985) realizaron una minuciosa descripción morfológica y fisiológica de estos Phomopsis, a los que denominaron $P$. longicolla, distinguiéndolos de aquellos previamente identificados como P. sojae sin. P. phaseoli var. sojae (Pps). Además, el teleomorfo (fase sexual, perfecta o peritecial) de Plo hasta el momento no ha sido observado (Hobbs et al., 1985; Vidić, Jasnic, \& Miladinovic, 1995; Pioli \& Morandi, 2005).

El ciclo biológico de Plo y Pps genera y dispone de diferentes fuentes de inóculo, reiniciando sus ciclos de parasitismo-saprofitismo en el rastrojo, las semillas y otros hospedantes. $P$. longicolla y D. phaseolorum var. sojae (Dps), (teleomorfo de $P p s$ ) fueron aislados de plantas de las Familias Fabaceaea (Vigna unguiculata, Phaseolus vulgaris, P. limensis, Lupinus spp., Arachis hypogaea y Glycine), Liliaceae (Allium cepa A. sativum), Solanaceae (Capsicum frutescens, Lycopersicon esculentum) y Umbeliferae (Foeniculum vulgare) (Udayanga et al., 2011; Santos \& Phillips, 2009; Santos, Vrandecic, Cosic, Duvnjak, \& Phillips, 2011; Vidić, Jasnić, \& Petrović, 2011) entre otras. La plasticidad biológica de Phomopsis spp. favoreció su expansión a diferentes agro-ecosistemas, afectando la calidad de semillas en especies arbóreas e incrementando el riesgo epidemiológico (Vidić et al., 1995).

En Argentina, cuando los ciclos de floración de Schinopsis balansae (quebracho colorado) coincidieron con períodos de abundantes 
precipitaciones se observó mayor incidencia de patógenos (entre ellos Phomopsis) y reducción en la germinación de las semillas (Alzugaray, Carnevale, Salinas, \& Pioli, 2007). Sin embargo, mientras en bosques nativos continuos se obtuvieron semillas contaminadas (no infectadas) por pocas especies fúngicas y alto porcentaje de germinación, en ecosistemas donde coexisten bosques con lotes de producción agrícola, la micota asociada a semillas forestales mostró índices de riqueza significativos y mayor diversidad de géneros fúngicos, incluyendo biocontroladores, celulolíticos, ligninolíticos y potenciales patógenos (Alzugaray, Carnevale, Salinas, \& Pioli, 2007).

La variabilidad genética en Plo y Pps evidenciada en estudios moleculares (Zhang, Riccioni, Pedersen, Kollipara, \& Hartman, 1998) ha promovido la aparición de nuevas variantes y razas fisiológicas que incrementaron la plasticidad parasítica, exponiendo al sistema de cultivos a riesgos epidemiológicos (Pioli \& Morandi, 2005).

En este contexto, la hipótesis de trabajo fue que en Argentina también existe variabilidad en la morfología y en el perfil molecular de los aislamientos de Diaporthe Phomopsis causales del Tizón de tallo, frutos (vainas) y semillas de G. max. Por ello es necesario una caracterización sistemática y rigurosa de las colecciones fúngicas disponibles con el fin de optimizar la conservación de su biodiversidad e inferir los mecanismos de variabilidad genética y su capacidad parasítica en diferentes hospedantes. El objetivo general de este trabajo consistió en validar la identidad y evaluar las relaciones biológicas de 12 aislamientos de Plo y $D p s$ obtenidos en distintos agro-ambientes templados y subtropicales de Argentina, para interpretar los mecanismos de variabilidad y aplicar estrategias de conservación de la biodiversidad fúngica. Los objetivos específicos fueron: 1) caracterizar morfológicamente aislamientos de Plo y Dps causales del TTVyS de G. max obtenidos de diferentes agro-ambientes; 2) validar molecularmente la identidad del grupo de aislamientos del complejo DP en estudio y sus controles.

\section{MATERIALES Y MÉTODOS}

Obtención de los aislamientos fúngicos: Los aislamientos de Plo, Pps y sus controles $D$. phaseolorum var. caulivora $(D p c)$ y $D$. phaseolorum var. meridionalis (Dpm) fueron obtenidos a partir de tallos, vainas y semillas sintomáticas de diferentes cultivares de G. max, expuestas a diversos agro-ambientes de Argentina. Las porciones de tejido fueron desinfectadas superficialmente con hipoclorito de sodio al $1 \%$ durante 45 a 60 segundos, sembradas en placas de Petri con medio Agar Papa Glucosado Acidulado (APGA), con $2 \%$ de glucosa y $1 \%$ de ácido láctico al $25 \%$. Las placas se incubaron en estufa a $26 \pm 1{ }^{\circ} \mathrm{C}$ durante siete días para promover desarrollo de colonias y una vez obtenidas, se realizaron repiques o transferencias de cada una de ellas a tubos con medio solidificado en plano inclinado (Vidić et al., 1995; Pioli et al., 2000). Los cultivos puros se conservaron a $4{ }^{\circ} \mathrm{C}$ (Fig. 1).

Caracterización morfológica de los aislamientos fúngicos: En el presente estudio se evaluaron 16 aislamientos de interés (diez Plo, dos $D p s$ y como controles dos $D p m$ y dos $D p c$ ) disponibles en la colección fúngica (Cuadro 1). Para su reactivación se tomaron pequeñas muestras de micelio $\left(1 \mathrm{~mm}^{2}\right)$ y se sembraron, por triplicado, en placas de Petri con medio APGA. Las colonias fúngicas se incubaron en las condiciones descritas y una vez desarrolladas, se trasladaron a una cámara de crecimiento con similar rango térmico y una alternancia de $12 \mathrm{hr}$ de luz-oscuridad, a fin de promover el desarrollo de estructuras fructíferas asexuales (picnidios) y/o sexuales (peritecios) (Pioli et al., 2003).

Una vez obtenidas las colonias puras y las fructificaciones asexuales y sexuales se analizaron comparativamente con base en sus características macro-morfológicas: color de las colonias, presencia y forma del estroma, presencia y tipo de picnidios y peritecios; y micro-morfológicas: tamaño y forma de conidios, ascos y ascosporas. La identidad de los hongos fue verificada de acuerdo a los trabajos previos de Vidić et al. (1995), Fernández y 


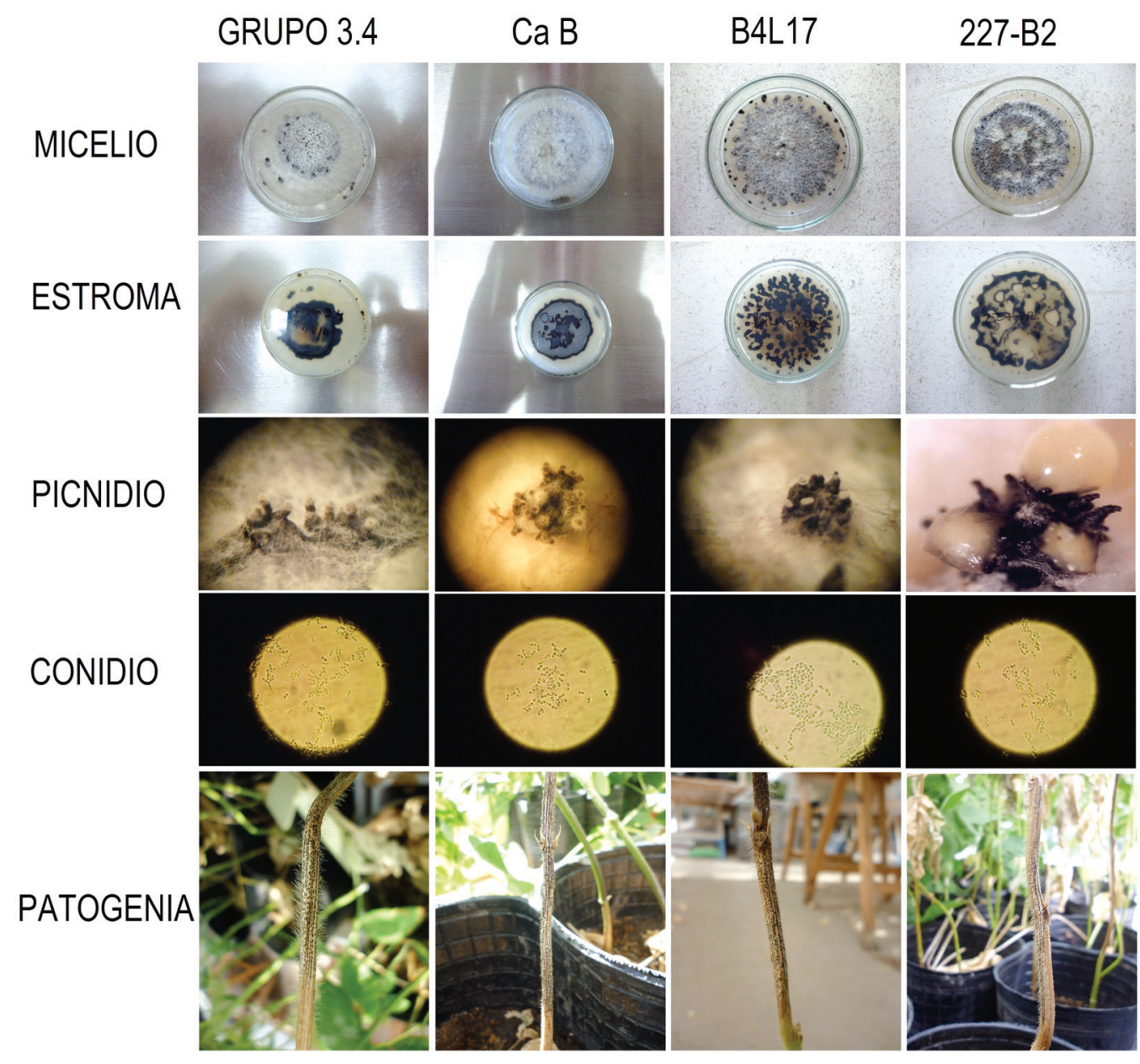

Fig. 1. Morfología de las colonias, síntomas y signos causados por Phomopsis longicolla, agente causal de la enfermedad Tizón del tallo y vaina.

Fig. 1. Morphology of colonies, symptoms and signs produced by Phomopsis longicolla, a causal agent (among others) of soybean stem and pod blight disease.

Hanlin (1996) y Pioli et al. (2003). Para ello, se usó un microscopio estereoscópico o lupa (CETI, Belgium, 40X) y un microscopio óptico (CETI, Belgium, 400 y 1000X). La validación de la identidad de los 16 aislamientos DP en estudio (Cuadro 1) se realizó por el registro triplicado ( $\mathrm{n}=48$ placas de cultivo) de la presencia o ausencia de 39 características o marcadores morfológicos relacionados con la macro y micro morfología de los hongos DP (Pioli et al., 2003) y un marcador asociado a la expresión fenotípica de la enfermedad (Hernández, 2014). Los aislamientos de DP identificados, luego fueron depositados y codificados en el Centro de Referencia en Micología (CEREMIC) y preservados duplicados en la Micoteca de la Sub Área Biodiversidad Vegetal y Microbiana (BioVyM) de la FCA.UNR.

Las observaciones registradas se volcaron a una planilla confeccionada con 16 muestras fúngicas y 39 variables (35 marcadores morfológicos + cuatro fenotípicos de la interacción). Estos resultados fueron analizados por un criterio de agrupamiento (cluster) basado en las distancias calculadas por Coeficiente de similitud de Jaccard y método de encadenamiento 
CUADRO 1

Aislamientos de Diaporthe Phomopsis incluidos en el estudio

TABLE 1

Diaporthe Phomopsis isolates included in the study

\begin{tabular}{|c|c|c|c|}
\hline \multicolumn{2}{|c|}{ Código de aislamiento ${ }^{a}$} & Especie o variedad & Localización geográfica \\
\hline AFP.Gpo $3.4^{\mathrm{b}}$ & $\mathrm{H} 1$ & Plo * o & Nogoyá, Entre Ríos \\
\hline AFP.Gpo $3.5^{\mathrm{b}}$ & $\mathrm{H} 2$ & $\mathrm{Plo}^{\circ}$ & Conc. del Uruguay, Entre Ríos \\
\hline AFP.Gpo $4.1^{\mathrm{b}}$ & $\mathrm{H} 3$ & $\mathrm{Plo}$ * & Conc. del Uruguay, Entre Ríos \\
\hline AFP.Gpo $4.3^{\mathrm{b}}$ & $\mathrm{H} 4$ & $\mathrm{Plo}^{\circ}$ & Conc. del Uruguay, Entre Ríos \\
\hline AFP.Gpo $4.4^{\mathrm{b}}$ & H5 & $\mathrm{Plo}^{\circ}$ & Conc. del Uruguay, Entre Ríos \\
\hline AFP.CaA & H6 & $\mathrm{Plo}^{\circ}$ & Cañizares, Tucumán \\
\hline AFP.CaB & $\mathrm{H} 7$ & $\mathrm{Plo}$ * o & Cañizares, Tucumán \\
\hline AFP.B5L16 & $\mathrm{H} 8$ & Plo * o & Firmat, Santa Fe \\
\hline AFP.B4L17 & H9 & Plo * o & Firmat, Santa Fe \\
\hline AFP.227-B2 & $\mathrm{H} 10$ & Plo * o & Ibarlucea, Santa Fe \\
\hline AFP.8413 & H11 & $D p s^{?} *$ o & Tucumán \\
\hline AFP.Qco7 & $\mathrm{H} 12$ & $D p s *$ о & Ceres, Santa Fe \\
\hline AFP.Dpc13 & H13 & $D p c$ & Zavalla, Santa Fe \\
\hline AFP.Dpc16 & H14 & $D p c *$ o & Esperanza, Santa Fe \\
\hline AFP.CE109 & H15 & $D p m *$ o & San Justo, Santa Fe \\
\hline AFP.CE112 & H16 & $\mathrm{Dpm}^{\mathrm{o}}$ & Clarcke, Santa Fe \\
\hline
\end{tabular}

a Plo: P. longicolla, Dps: D. phaseolorum var. sojae, Dpc: D. phaseolorum var. caulivora, Dpm: D. phaseolorum var. meridionalis. Colección obtenida e identificada con base en marcadores morfológicos en el marco de PID.AGR-100 y PID. AGR-185 (Director. R.N. Pioli 2007-14).

${ }^{\mathrm{b}}$ Aislamientos obtenidos y aportados por la Ing. Agr. N. Formento, EE INTA Paraná, E. Ríos. Conc.: Concepción del Uruguay, E. Ríos.

${ }^{\circ}$ Aislamientos usados en la Caracterización Molecular.

* Aislamientos usados en las Pruebas de Patogenicidad.

promedio con el programa estadístico InfoStat (Pioli et al., 2003).

Validación de la identidad por caracterización molecular: La caracterización molecular se realizó combinando dos técnicas basadas en la reacción en cadena de la polimerasa (PCR) y el uso de varios oligonucleóticos (primers) de diferente especificidad.

El análisis por ITS (Internal transcriptional Sequences) se desarrolló utilizando el oligonucleótico o primer ITS4 (5' TCCTCCGCTTATTGATATGC 3`). Y el análisis realizado mediante la amplificación del oligonucleótido PL5, específico de Plo, se realizó con dos cebadores: un directo (5' CGAGCTCGCCACTAGATTTCA 3') y un reverso (5' CCTCAAGCCTGGCTTGGTGATGG 3'). Para ambos procesos, las mezclas de reacción se obtuvieron en un $\mathrm{Vf}=25 \mu \mathrm{L}$, y las amplificaciones fueron realizadas en un termociclador
Bio-Rad-T100 con una etapa preliminar de dos min a $96{ }^{\circ} \mathrm{C}$, una etapa de 35 ciclos de un min a $96{ }^{\circ} \mathrm{C}$, un min a $55^{\circ} \mathrm{C}$, y dos min a $72{ }^{\circ} \mathrm{C}$; y una etapa final de diez min a $72{ }^{\circ} \mathrm{C}$ (Zhang et al., 1998).

El análisis por RAPDs (Randomly Amplified Polymorphic DNAs, análisis de polimorfismos por la amplificación al azar del ácido desoxirribonucleico genómico) fue desarrollado con dos cebadores decámeros arbitrarios OP-AA01 (5'AGACGGCTCC3') y OP-AA06 (5'GTGGGTGCCA3') (Operon Technologies, USA) utilizados en trabajos previos. Las mezclas de reacción de un Volumen final (Vf) de $25 \mu \mathrm{L}$ estuvieron compuestas de: $5 \mathrm{mM}$ de cada dNTP $(1 \mu \mathrm{L}) ; 5 \mu \mathrm{L}$ de Go Taq ${ }^{\circledR}$ Reaction Buffer, $4.5 \mu \mathrm{L}$ de primer Xng $/ \mu \mathrm{L} ; 0.25 \mu \mathrm{L}$ de AmpliTaq ácido deoxiribonucleico Polimerasa (5 unidades $/ \mu \mathrm{L}$ ) (Fernández \& Hanlin, 1996; Pioli et al., 2003). Las amplificaciones fueron realizadas en un termociclador Bio-Rad-T100 
con una etapa preliminar de tres min a $94{ }^{\circ} \mathrm{C}$ una etapa que consta de 40 ciclos de un min a $94{ }^{\circ} \mathrm{C}$, un min a $35{ }^{\circ} \mathrm{C}$ y dos $\min$ a $72{ }^{\circ} \mathrm{C}$. Finalmente, una vez realizado los 40 ciclos, se procedió a una etapa final de diez min a $72{ }^{\circ} \mathrm{C}$ (Gally, Ramos, Dokmetzian, \& Lopez, 2007).

\section{Extracción y amplificación del ácido} desoxirribonucleico (ADN) genómico fúngico: Los aislamientos de D/P (Plo, Pps, Dpm y $D p c$ ) incluidos en este estudio se han indicado en el cuadro 1. Los aislamientos se cultivaron en placas de Petri con medio APGA y se incubaron a $26 \pm 2{ }^{\circ} \mathrm{C}$ en oscuridad de acuerdo a lo descrito. Luego de cinco días, el micelio de cada uno de los aislamientos fue recolectado con escalpelo (uno a cinco $\mathrm{mg}$ de tejido fresco) en microtubos de centrífuga de $1.5 \mathrm{~mL}$ (Pioli et al., 2003). La extracción del ADN genómico fúngico total fue realizado de acuerdo al protocolo indicado para uso del kit de extracción Wizard ${ }^{\circledR}$ Genomic DNA. La integridad del ADN genómico de cada uno de los aislamientos se evaluó por la presencia de una banda de alto peso molecular, mediante electroforesis en geles de agarosa al $1 \%$.

Resolución en geles de poliacrilamida y análisis de los datos moleculares: La separación de los fragmentos de amplificación se realizó en geles de poliacrilamida $(6 \% \mathrm{p} / \mathrm{v})$. Los geles se armaron en celdas Sequi-Gen GT Sequencing (Bio-Rad Laboratories). Se usaron marcadores (Biodynamics) de peso molecular con incrementos de 50-100 pares de bases). La visualización de marcadores moleculares se realizó de acuerdo al manual técnico del "DNA Silver Staining System" (Promega) para tinción de geles de secuencia con nitrato de plata (nitrato de plata $0.1 \% \mathrm{p} / \mathrm{v}$, formaldehído $0.07 \% \mathrm{v} / \mathrm{v})$. El revelado se realizó con dos litros de solución de carbonato de sodio (carbonato de sodio $3 \% \mathrm{p} / \mathrm{v}$, formaldehído 0.07 $\% \mathrm{v} / \mathrm{v}$, tiosulfato de sodio $0.4 \% \mathrm{p} / \mathrm{v}$ ) hasta la aparición de las bandas, y se detuvo la reacción con ácido acético $10 \% \mathrm{p} / \mathrm{v}$. Los patrones de bandas se analizaron directamente en los vidrios sobre un transiluminador de luz blanca (Gally et al., 2007).

A partir del perfil de bandas obtenido en el gel de poliacrilamida, se construyó una matriz de datos basada en la presencia/ausencia (uno: presencia de banda; cero: ausencia de banda; cinco: dudoso) de cada una de esas bandas para todos los aislamientos en estudio. Esta matriz fue utilizada para realizar el análisis fenético de los aislamientos por un criterio de agrupamiento o cluster basados en el grado de similitud. Se aplicó el método de resolución de Ward basado en distancias Eucledias promedio (INFOSTAT) con base en trabajos previos (Pioli et al., 2003; Gally et al., 2007). Los dendrogramas generados por la amplificación con cada uno de los oligonucleótidos y un dendrograma integrado obtenido a partir de la amplificación de los cuatro oligonucleótidos (OPA1, OPA6, ITS4 y PL5) (Fig. 4). indicaron el porcentaje de similitud de las cepas DP evaluadas, con el respectivo porcentaje de encadenamiento (Hernández, 2014).

\section{RESULTADOS}

Caracterización morfológica: Los síntomas típicos de cada interacción planta-patógeno (Tizón o Cancro) y las estructuras fúngicas típicas de $P l o, D p s$ y los controles $D p m$ y $D p c$ se desarrollaron aproximadamente a los 30-35 días posteriores a la inoculación de cada aislamiento DP. De esta manera, el tipo de lesión o expresión fenotípica de la enfermedad causada en los genotipos de soja por cada uno de las cepas, fue incorporada como un marcador morfológico adicional (Fig. 1). para el análisis fenético de similitud y la obtención del dendrogama respectivo (Fig. 2).

\section{Aislamientos causantes de Tizón de tallos y vainas de soja (TTVyS):}

A.a- P. longicolla: los aislamientos identificados como Plo produjeron micelio blanquecino que puede cambiar con la edad, tornándose a una colonia con tintes amarillo-verdoso y grisáceos. Los picnidios, de aspecto costroso e irregular, desarrollaron frecuentemente agrupados, 

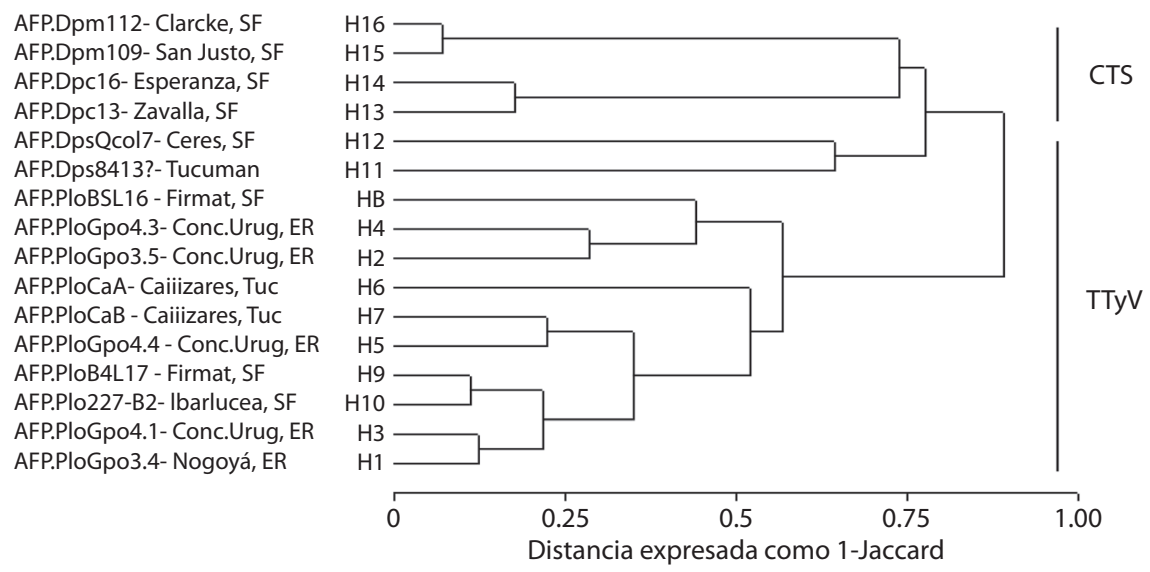

Fig. 2. Dendrograma basado en el análisis de similitud de 16 aislamientos de Diaporthe-Phomopsis obtenido del análisis de 48 muestras fúngicas y 39 marcadores morfológicos. (*) CTS: Fenotipo de Cancrosis del Tallo y TTyV: Fenotipo de Tizón del tallo y vaina de soja.

Fig. 2. Dendrogram based on the similarity analysis of 16 Diaporthe - Phomopsis isolates, obtained from 48 fungal replicates and 39 morphological markers. $\left({ }^{*}\right)$ SSC: Soybean stem canker, and BSP: Blight of Stem and Pods phenotypes.

en encadenamientos extendidos y ocasionalmente solitarios. Mostraron un cuello o pico $>400 \mu \mathrm{m}$ largo y exudaron sólo $\alpha$ conidios como señala la bibliografía para esta especie. Los estromas fueron oscuros y frecuentemente extendidos desde el centro de la colonia hacia los márgenes y algunos segmentados. Cuando se los inoculó en G. max mostraron síntomas y signos asociados al TTVyS (Fig. 1).

A.b- D. phaseolorum var. sojae (anamorfo $P$. phaseoli var sojae): los aislamientos identificados como Pps presentaron micelio generalmente flocoso, formando cordones y de diferentes tonalidades desde blanco, blancogris, blanco-beige, gris claro, gris-amarronado, ocasionalmente con tintes blanco-amarillento. Los picnidios fueron pequeños, usualmente múticos o con muñón corto $(<400 \mu \mathrm{m})$, solitarios, apareados o adyacentes. Los estromas de Pps son variables, disgregados, dispersos, en arreglos concéntricos o ausentes. Pps produce $\alpha$ conidios (ovoides), ó $\beta$ (filiformes) ó ambos tipos de conidios $(\alpha$ y $\beta$ ) como fue observado en el aislamiento Pps-AFP.Qcol7. La presencia de $\beta$ conidios es la que separa morfológicamente las cepas de Pps de las pertenecientes a Plo.
En aquellos aislamientos donde los caracteres estromáticos y conidiales no fueron suficientemente definidos como el AFP.8413, la identidad fue finalmente determinada por marcadores moleculares. No obstante, ambos Pps identificados provisoriamente por su morfología, produjeron síntomas y signos de TTVyS sobre G. max. En la naturaleza, los aislamientos Pps asociados a los restos de cosecha se hibridan y producen fructificaciones sexuales o peritecios de Dps (forma perfecta o teleomorfa). Sin embargo, los aislamientos desarrollados in vitro no produjeron peritecios, manifestando su condición biológica de heterotalismo.

Aislamientos DP usados como controles
experimentales:
B.a- $D$. phaseolorum var. caulivora: Los
aislamientos Dpc mostraron micelio blanque-
cino con tintes amarillentos. Algunos pocos se
tornan de color marrón claro u ocráceo con la
edad. La producción de picnidios es esporádica
y escasa. A medida que el cultivo envejece,
se forman vesículas estromáticas inicialmente
pequeñas y circulares, de color marrón a negro,
luego irrumpentes y dispersas en el medio. La


condición de homotalismo de $D p c$, hace que en las colonias asexuales de $P p c$, las hifas (n) se fusionen $\mathrm{y}$ originen fructificaciones sexuales o peritecios de $D p c$. Estos se pueden observar en grupos y con los picos emergentes como en un manojo. Cuando los aislamientos $D p c$ fueron inoculados mostraron síntomas y signos de CTS.

B.b- D. phaseolorum var. meridionalis: Los aislamientos de Dpm produjeron colonias con micelio distribuido en cordones aplanados, que inicialmente son blanquecinas y luego se tornan de color bronceado y tintes gris-amarronados. Los aislamientos originaron colonias de Ppm (fase asexual) que produjeron picnidios con $\alpha$ conidios. Por su condición de homotalismo, y posteriormente a la fusión de hifas (n), se produjeron peritecios sexuales de Dpm, solitarios, con cuello o pico delgado y elongado. El reverso de las colonias mostró áreas de tonos beige, tostado a amarronado, no presentando estromas definidos. Ocasionalmente estas colonias mostraron áreas difusas más oscuras de forma irregular formadas por hifas de resistencia, de acuerdo a lo informado en trabajos previos (Pioli et al., 2003). Cuando los aislamientos Dpm fueron inoculados en $G$. max desarrollaron síntomas y signos de CTS.

La Fig. 2 muestra el dendrograma obtenido al analizar el grado de semejanza promedio de los 16 aislamientos DP $(n=48)$, y 39 marcadores morfológicos. El análisis fenético permitió agrupar a las cepas fúngicas aisladas en las cuatro taxas de DP (Pps, Plo y controles Dpm y $D p c$ ) incluidas en el complejo DiaporthePhomopsis asociado a G. $\max$ (Fig. 2), con una correlación cofenética de 0.724 . Este valor indica un adecuado ajuste entre el dendrograma obtenido y los datos ingresados para su construcción.

El dendrograma diferenció dos grupos principales G.I y G.II relacionados con una distancia de 0.90 y sólo $10 \%$ de similitud. El G.I incluyó a los aislamientos del binomio Diaporthe-Phomopsis causales de CTS (Dpm y $D p c$ ), usados como controles experimentales, $\mathrm{y}$ a los dos aislamientos de Dps (anamorfo: Pps), causales de TTVyS, con una distancia de $0.77 \mathrm{y}$
$23 \%$ de similitud. El análisis mostró, además, que las dos variedades intra-específicas de $D$. phaseolorum, Dpm y $D p c$, causales de CTS, se ubicaron a 0.75 de distancia, indicando una similitud morfológica del $25 \%$. El análisis intra-varietal en Dpm ubicó a sus aislamientos a 0.06 de distancia ó $94 \%$ similitud y los de $D p c$ a 0.16 y $84 \%$ similitud. Por su parte, los aislamientos $D p s$, se ubicaron a una distancia de 0.63 y $37 \%$ de similitud morfológica.

El G.II incluyó sólo a los aislamientos de Plo causales de TTVyS, mostrando un rango de distancia intra-específica entre 0.10 y 0.56 equivalente a 90 y $44 \%$ de similitud, respectivamente. Las variedades Dpm y $D p c$ se diferenciaron morfológicamente por la producción de picnidios y alfa conidios, por la distribución de peritecios y longitud de ascos y ascosporas. Sin embargo, es importante destacar que ambas variedades causan una enfermedad similar denominada CTS. Los aislamientos evaluados de la variedad $D p s$ presentaron algunas características como textura de micelio, color de colonia, presencia y distribución de estromas y picnidios múticos que los han aproximado en similitud a $D p m$ y $D p c$ y distanciado de Plo. Este resultado basado en caracteres morfológicos revalida y fortalece la pertenencia de la var. sojae, junto a las variedades meridionalis y caulivora, a la especie D. phaseolorum-P. phaseoli.

$\mathrm{Al}$ analizar la relación entre similitud morfológica y ambiente agro ecológico del cual fueron obtenidos cada uno de los aislamientos, se observó que no existe una relación entre estas dos variables (Fig. 2).

Por otra parte, respecto a la relación entre similitud morfológica y la expresión fenotípica de la dos patologías (TTVyS y CTS) causadas por el complejo DP, se observó que individuos disímiles morfológicamente y pertenecientes a dos especies distintas pueden causar, de manera asociada, la misma o similar patología. Por ejemplo, D. phaseolorum var. sojae y la especie independiente $P$. longicolla se asocian y causan la enfermedad de final de ciclo: el Tizón del Tallo y Vaina de la soja (Fig. 2). 

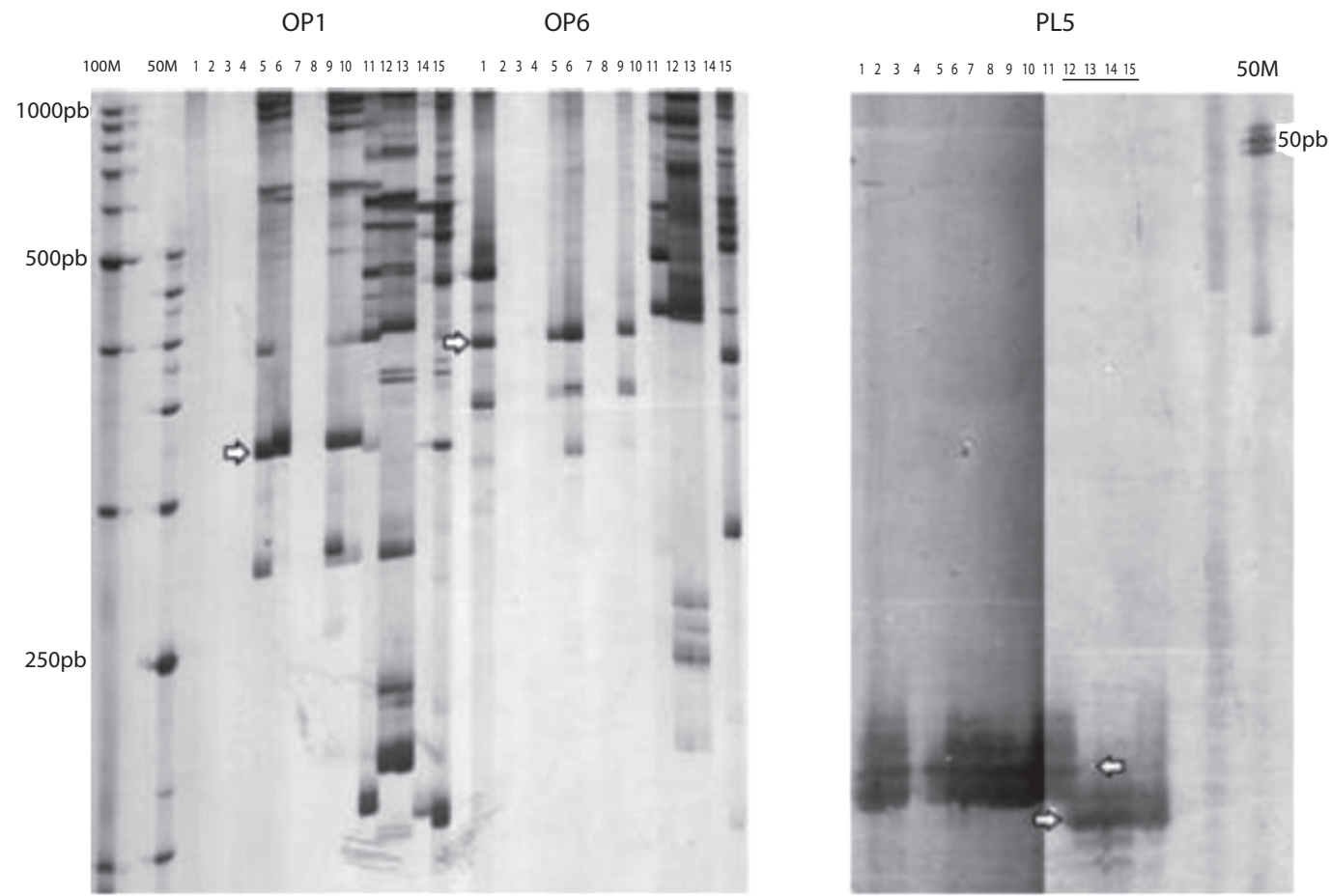

Fig. 3. (A) Perfil de bandas de Phomopsis longicolla (Plo) y sus controles obtenido a partir de amplicones de los oligonucleótidos OPA1 y OPA6; (B) Perfil de bandas específicas para Plo obtenido a partir de amplicones del oligonucleótido Plo5. M: Marcador de Peso Molecular con incrementos de 50-100 pares de bases.

Fig. 3. (A) Marker profile of Phomopsis longicolla (Plo) and their controls, obtained from amplycons of OPA1 and OPA6 primers. (B) Marker profile of Phomopsis longicolla (Plo) and their controls, obtained from amplycons of Plo5 specific primer. M: Molecular weight marker based on 50-100 pb increases.

Caracterización molecular: El perfil de bandas generado en gel de poliacrilamida por los oligonucleótidos OPA1 y OPA6 (Fig. 3) y el análisis de polimorfismos logrado a partir de RAPD mostró un $100 \%$ de polimorfismo distribuido al azar en el genoma fúngico a nivel inter-específico ( $P$. longicolla y $P$. phaseoli) e intra específico ( $P$. phaseoli var. sojae, var. meridionalis y var. caulivora). El dendrograma parcial (no mostrado) generado a partir de las amplificaciones con dichos cebadores agrupó a nueve de las diez cepas propuestas por morfología como Plo (el grupo 4.1 no amplificó), anexando además al aislamiento AFP.8413 (ubicado por su morfología y provisoriamente como Dps/Pps (Cuadro 1). De esta manera, el AFP.8413 fue re-ubicado en el taxón correspondiente a Phomopsis longicolla. Luego el taxón Plo se unió, con diferente grado de similitud, a las tres variedades $D p s, D p c$ y $D p m$ incluidas en la especie Diaporthe phaseolorum (anamorfo o asexual P. phaseoli), completando los cuatro taxones pertenecientes al Complejo Diaporthe-Phomopsis.

Asimismo, el dendrograma parcial (no mostrado) generado por los oligonucleótidos ITS4 y PL5 agrupó los aislamientos Plo propuestos por morfología en un único grupo. No obstante, seis Plo fueron relacionados estrechamente con $100 \%$ de similitud: AFP.Grupo 4.4, AFP.Grupo 3.5, AFP.Grupo 4.3, AFP.Grupo 3.4, AFP.B5L16 y AFP.B4. A quienes se unieron el AFP.8413 (re ubicado como Plo), el AFP.227B2 y dos Plo de Tucumán (AFP. $\mathrm{CaA}$, AFP.CaB). Finalmente, se anexó el H11QCol7 (referente de Dps) junto a H5-CaA y H6-CaB (identificados como Plo). La similitud observada en el patrón de pocas bandas 
observadas entre Plo y Pps en geles generados por ITS 4, ha sido observada, además, en estudios previos que incluyeron a Plo y las tres variedades de $D$. phaseolorum ( $P$. phaseoli) debido a que esos oligonucleótidos amplifican zonas conservadas del genoma principalmente entre taxones estrechamente relacionados a nivel inter e intra-específica. Por su parte, el oligonucleótido específico Plo5 diferenció el perfil de banda específica de Plo amplificada y observada en las nueve cepas Plo + H10-8413, de aquellos pertenecientes a las tres variedades de D. phaseolorum / P. phaseoli (Pps, Ppm y Ppc) (Fig. 3B).

El análisis independiente de polimorfismos logrados a partir de ITS4 mostró un $100 \%$ de bandas polimórficas pero sin discriminar entre las especies P. longicolla y P. phaseoli. Ya que diferenció a los aislamientos de acuerdo a su patogenia, de esta manera separó a aquellas cepas causales de Tizón del tallo y vaina de $G$. $\max (P$. longicolla y $P$. phaseoli var. sojae) de aquellas causales de Cancrosis del tallo ( $P$. phaseoli var. meridionalis y var. caulivora). Mientras, que el análisis a partir del oligonucleótido específico Plo5 mostró $100 \%$ de polimorfismos a nivel inter específico ( $P$. longicolla y $P$. phaseoli) pero no detectó polimorfismos intra específicos o entre las variedades de $P$. phaseoli (var. sojae, var. meridionalis y var. caulivora).

De manera complementaria e integradora, la Fig. 4 muestra el dendrograma generado a partir de 255 bandas o marcadores moleculares resueltos por los cuatro oligonucleótidos (OPA1, OPA6, ITS4 y PL5). A partir del análisis, se obtuvieron las distancias Euclideas intra e inter nodos y la Correlación cofenética de 0.992 .

Desde el punto de vista de la biología fúngica, el dendrograma diferenció a cuatro grupos principales obtenidos a partir de los amplicones polimórficos de cuatro oligonucleótidos ITS4, PL5, OPA1 y OPA6 (Fig. 4).

El grupo G.I incluyó a los diez aislamientos de Plo distribuidos en tres sub grupos: G.IA fue integrado por cinco cepas de Plo (AFP.Grupo 3.5, AFP.Grupo 4.3, AFP.Grupo 3.4, AFP.B4L17 y AFP.B5L16) ubicados entre 0.06 y 0.08 DE y con 94 y $92 \%$ de similitud, respectivamente. Este grupo reunió dos aislamientos de Concepción del Uruguay y uno de Nogoyá de la Pcia. de Entre Ríos (325' S - 59 $1^{\circ}$ W), con dos cepas de Firmat, Pcia. de Santa Fe $\left(33^{\circ} 5^{\prime} \mathrm{S}-61^{\circ} 5^{\prime} \mathrm{W}\right)$ (Cuadro 1 y Fig. 4). A este clado se unió el AFP.8413, Pcia. Tucumán $\left(26^{\circ} 7^{\prime} \mathrm{S}-65^{\circ} 2^{\prime} \mathrm{W}\right)$ con $67 \%$

Dendrograma obtenido por amplificación por OPA1, OPA6, ITS y PL5

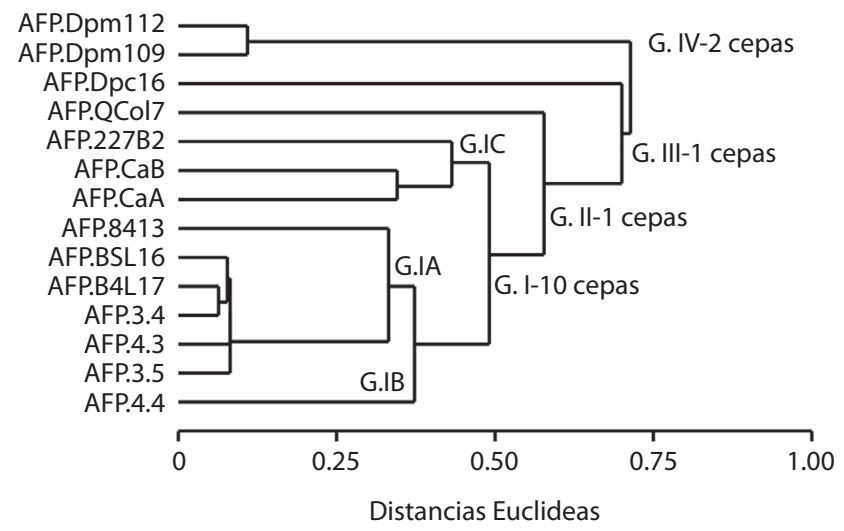

Fig. 4. Dendrograma basado en el análisis de similitud de 14 aislamientos de Diaporthe-Phomopsis (10 Plo, 1 Dps, 2 Dpm y $1 D p c$ ) por amplificación de los oligonucléotidos OPA1, OPA6, ITS4 y PL5 y resolución de un perfil de 255 bandas.

Fig. 4. Dendrogram based on the similarity analysis of 14 Diaporthe - Phomopsis isolates (10 Plo, 1 Dps, 2 Dpm y 1 Dpc), obtained from OPA1, OPA6, ITS4 y PL5 primers and 255 markers. 
de similitud y 0.33 DE y por ello, fue re ubicado taxonómicamente como perteneciente a la especie Plo. Asimismo, el G.IA se asoció el G.IB que incluyó a un único aislamiento Plo, el AFP.Grupo 4.4 de Concepción del Uruguay (Pcia. de Entre Ríos) relacionados con $0.37 \mathrm{DE}$ y $63 \%$ de similitud. Mientras que el G.IC fue integrado por tres cepas de Plo, el AFP.CaA y AFP.CaB de Cañizares, Pcia. de Tucumán $\left(26^{\circ} 7^{\prime} \mathrm{S}-65^{\circ} 2^{\prime} \mathrm{W}\right)$ que se ubicaron a $0.35 \mathrm{DE}$ con $65 \%$ de similitud. Y finalmente se anexó el Plo AFP.227B2 de Ibarlucea, Pcia. Santa Fe $\left(32^{\circ} 9^{\prime} \mathrm{S}-60^{\circ} 8^{\prime} \mathrm{W}\right)$ se unió con este grupo de Plo a los 0.43 DE y $57 \%$ de similitud.

El grupo G.II fue constituido por una única cepa, la AFP.Qcol7 perteneciente a Dps-Pps y relacionada a 0.58 unidades de DE y $42 \%$ de similitud (Cuadro 1 y Fig. 4). El grupo G.III, por su parte, también estuvo constituido por una cepa de $D p c$, la AFP.Dpc16, que mostró 0.70 unidades de DE y $30 \%$ de similitud con el resto de aislamientos DP (Cuadro 1 y Fig. 4).

Por su parte, el grupo G.IV comprendió dos cepas de Dpm, las AFP.Dpm109 y la AFP. Dpm112, con una DE intra-varietal de 0.11 y $89 \%$ de similitud. Mientas la DE inter-varietal y específica con el resto de los aislamientos DP fue de 0.71 DE y $29 \%$ similitud (Cuadro 1 y Fig. 4).

Finalmente, desde el punto de vista patogénico, los grupos G.I (Plo) y G.II (Dps) incluyeron aislamientos causales de TTVyS. Y los grupos G.III $(D p c)$ y G.IV $(D p m)$ agruparon a los aislamientos causales de CTS (Fig. 2) incluidos en el presente estudio como controles experimentales.

\section{DISCUSIÓN}

Las cualidades macro-morfológicas referidas a textura y color de colonias (anverso y reverso), forma y distribución de estromas, desarrollo, forma y distribución de picnidios y peritecios, y los caracteres micro-morfológicos como tamaño y forma de conidios, ascos $\mathrm{y}$ ascosporas permitieron agrupar los nuevos aislamientos patogénicos de G. max en los taxones conocidos (Plo y $D p s, D p c$ y $D p m$ ) del complejo D/P. Los resultados obtenidos en el presente análisis mostraron una tendencia de agrupamiento bio-morfológica similar a estudios previos propios (Pioli, Morandi, \& Bisaro, 2001; Pioli, Morandi, \& Luque, 2002; Pioli et al., 2003) y de otros autores (Fernández \& Hanlin, 1996; Santos et al., 2011; Vidić et al., 2011).

Adicionalmente, la caracterización morfológica basada en lo reportado por Fernández y Hanlin (1996), Pioli y Morandi (2005) y Vidić et al. (1995), permitió identificar como $P$. longicolla a tres nuevos aislamientos nóveles (AFP.B5L16, AFP.B4L17 y AFP.227-B2). Sin embargo, en el caso del aislamiento AFP8413, que presentó caracteres morfológicos no categóricos respecto a forma y distribución de picnidios, tipo de conidios, presencia y distribución de estromas, los marcadores morfológicos no fueron suficientemente claros para definir su pertenencia al taxón Dps-Pps. Por ello, la identidad del AFP8413 fue finalmente determinada por marcadores moleculares. Las tres variedades de $D$. phaseolorum (anamorfo P. phaseoli) Dpm, Dpc y $D p s$ producen peritecios en la naturaleza. No obstante en el presente estudio, sólo los aislamientos Ppm y Ppc (anamorfos) y por su condición biológica de homotalismo, desarrollaron peritecios -in vitrocorrespondientes a sus respectivos teleomorfos $D p m$ y $D p c$. Mientras los aislamientos de Pps (anamorfos) sembrados en medio de cultivo no originaron la fase sexual o teleomorfa de Dps ni formaron sus fructificaciones debido a su condición de heterotalismo (Pioli \& Morandi, 2005). Estas observaciones biológicas son coincidentes con el comportamiento mostrado por colecciones de DP de distinta constitución intra e inter específica y origen geográfico (datos no mostrados).

La caracterización molecular de 14 aislamientos DP (AFP.4.1 y el AFP.Dpc13 no amplificaron en gel de agarosa o el de poliacrilamida, respectivamente) se basó en el análisis integrado de 250 amplicones obtenidos por dos técnicas diferentes RAPDs e ITS y cuatro cebadores OPA1, OPA6, ITS4 y PL5, y agrupó a los aislamientos evaluados en los cuatro taxones 
de DP: Phomopsis longicolla y las tres variedades de Diaporthe phaseolorum (anamorfo P. phaseoli), siendo ellas, D. phaseolorum var. sojae, D. phaseolorum var. caulivora y D. phaseolorum var. meridionalis. Las distancias Euclideanas observadas intra e internodos correspondieron con un valor de Correlación cofenética de 0.983 considerado adecuado para este tipo de análisis (Gally et al., 2007).

La caracterización molecular realizada en este estudio validó la identidad de 13 de los 14 (ésto es el 92.5\%) de los aislamientos de DP caracterizados por marcadores morfológicos, entre ellos los tres aislamientos nóveles AFP. B5L16, AFP.B4L17 y AFP.227-B2. Asimismo y de manera superadora, el análisis molecular complementario corrigió las limitaciones derivadas de la aplicación de marcadores sólo morfológicos y logró reubicar al aislamiento AFP.8413 de identidad dudosa, en el nodo correspondiente a $P$. longicolla.

Asimismo la caracterización molecular puso en evidencia valores significativos de similitud en el genoma fúngico de aislamientos procedentes tanto de un área geográfica similar como de aquellas muy distantes.

El presente estudio logró definir la identidad de los aislamientos analizados y distribuirlos entre los cuatro taxones DP: diez aislamientos incluidos en Plo (Grupo 4.4, Grupo 3.5, Grupo 4.3, Grupo 3.4, CaA, CaB, B5L16, B4L17, 227B2, 8413), un aislamiento incluido en Dps (AFP.Qcol7), un aislamiento en $D p c$ (AFP.Dpc16) y dos aislamientos incluidos en Dpm (AFP.Dpm109 y AFP.Dpm112).

En este trabajo se demuestra la importancia de una caracterización sistemática y rigurosa en los niveles fenotípico y molecular de las colecciones de patógenos DP para evitar duplicaciones o identificaciones incorrectas. De esta manera, se promueve la conservación y/o preservación de su biodiversidad con base en una apropiada identificación y el conocimiento de aquellas estrategias biológicas (homo u heterotalismo), relacionadas con mecanismos de variabilidad genética fúngica y plasticidad para colonizar y ampliar el rango de hospedantes.

\section{AGRADECIMIENTOS}

Este trabajo fue realizado en el marco de los Proyectos PID.UNR-AGR100 y AGR185 (Director R. N. Pioli) financiados por la Secretaría de Ciencia y Técnica de la UNR, Sec. Estado de Ciencia, Tecnología e Innovación de Santa Fe, PRIETEC 2009 y Programa Estratégico Alimentario 2011. Agradecemos a Bibiana Ferrari por atender nuestras consultas.

\section{RESUMEN}

Diaporthe (telomorfo) - Phomopsis (anamorfo) (DP) constituye un grupo fúngico de amplia diversidad genética con más de 900 especies distribuidas en un amplio rango de hospedantes que incluye especies cultivadas y no cultivadas, forestales, frutales y malezas. Los aislamientos de DP son hemi-biótrofos y disponen de diferentes fuentes de inóculo primario, como el rastrojo y las semillas, para reiniciar sus ciclos de parasitismo-saprofitismo. Ellos colonizan los tejidos del hospedante desde los estadios tempranos del desarrollo y establecen relaciones nutricionales de endofitia y necrotrofia fúngica. La plasticidad del género Phomopsis ha favorecido su expansión a diferentes agroecosistemas y hospedantes constituyendo un importante riesgo epidemiológico. El objetivo fue validar la identidad y evaluar las relaciones biológicas de 12 aislamientos de $P$. longicolla y $D$. phaseolorum var. sojae obtenidos en distintos agro-ambientes templados y subtropicales de Argentina, para analizar la variabilidad y estrategias de conservación de la bio-diversidad fúngica. Las cualidades macro-morfológicas (textura y color de colonias, forma y distribución de estromas, desarrollo, forma y distribución de cuerpos fructíferos), y los caracteres micro-morfológicos (tamaño y forma de conidios, ascos y ascosporas) permitieron identificar a tres nuevos aislamientos de $P$. longicolla incluidos en el complejo D/P. El análisis molecular complementario corrigió las limitaciones derivadas de la caracterización basada sólo en marcadores morfológicos y logró reubicar al aislamiento AFP.8413 de identidad dudosa, en el nodo correspondiente a $P$. longicolla. De esta manera, la caracterización molecular definió la identidad de los aislamientos y los ubicó en los 4 taxones del complejo DP: diez aislamientos fueron incluidos en Plo (AFP.Gpo 4.4, AFP.Gpo 3.5, AFP.Gpo 4.3, AFP.Gpo 3.4, AFP.CaA, AFP. CaB, AFP.B5L16, AFP.B4L17, AFP.227B2, AFP.8413), un aislamiento incluido en Dps (AFP.Qcol7), un aislamiento en $D p c$ (AFP.Dpc16) y dos aislamientos incluidos en $D p m$ (AFP.Dpm109 y AFP.Dpm112). La adecuada identificación de $P$. longicolla y el avance en el conocimiento de las relaciones biológicas (hibridaciones homo o heterotálicas) entre variedades de $D$. phaseolorum ( $P$. phaseoli) y especies de Diaporthe - Phomopsis permiten comprender la plasticidad para colonizar un amplio rango de hospedantes, 
los mecanismos de variabilidad genética y la preservación de la diversidad fúngica.

Palabras claves: hongos hemi-biotrofos, Phomopsis longicolla, epidemiología, análisis de agrupamiento, agro-biología.

\section{REFERENCIAS}

Alzugaray, C., Carnevale, N. J., Salinas, A. R., \& Pioli, R. N. (2007). Factores bióticos y abióticos que afectan la calidad de las semillas de Schinopsis balansae y Aspidosperma quebracho-blanco. Revista Iberoamericana de Micología, 24(2), 142-147.

Ash, G. J., Stodart, B., Sakuanrungsirikul, S., Anschaw, E., Crump, N., Hailstones, D., \& Harper, J. D. (2010). Genetic characterization of a novel Phomopsis sp., a putative biocontrol agent for Carthamus lanatus. Mycologia, 102(1), 54-61.

Benavídez, R., Pioli, R. N., \& Morandi, E. N. (2010). Response of the Edamame edible soybean germoplasm to Diaporthe phaseolorum, causal agents of Soybean Stem Canker, in Argentina. Tropical Plant Pathology, 35(1), 1-11.

Crous, P. W., \& Groenewald, J. Z. (2005). Hosts, species and genotypes: opinions versus data. Australian Plant Pathology, 34, 463-470.

Debaeke, P., \& Moinard, J. (2010). Effect of crop management on epidemics of phomopsis stem canker (Diaporthe helianthi) for susceptible and tolerant sunflower cultivars. Field Crops Research, 115(1), 50-60.

Farr, D. F., Castlebury, L. A., \& Rossman, A. Y. (2002). Morphological and molecular characterization of Phomopsis vaccinii and isolates of Phomopsis from blueberry and cranberry in the eastern United States. Mycologia, 94(3), 494-504.

Fernández, F. A., \& Hanlin, R. T. (1996). Morphological and RAPD analyses of Diaporthe phaseolorum from soybean. Mycologia, 88, 425-440.

Gally, M., Ramos, A. M., Dokmetzian, D., \& Lopez, S. E. (2007). Genetic variability of Phytophthora sojae from Argentina. Mycologia, 99(6), 877-883.

Heller, A., \& Gierth, K. (2001). Cytological observations of the infection process by Phomopsis helianthi (Munt.Cvet) in leaves of sunflower. Journal of Phytopatho$\log y, 149,347-357$.

Hernández, F. (2014). Caracterización de la reacción de genotipos de Glycine max al interactuar con aislamientos de P. longicolla de diferentes agroecosistemas de Argentina (Tesina). Universidad de Morón, Argentina.
Hobbs, T. W., Schmitthenner, A. F., \& Kuter, G. A. (1985). A new Phomopsis specie from soybean. Mycologia, 77(4), 535-544.

Kleczewski, N. M., Bauer, J. T., Bever, J. D., Clay, K., \& Reynolds, H. L. (2012). A survey of endophytic fungi of switchgrass (Panicum virgatum) in the Midwest, and their putative roles in plant growth. Fungal Ecology, 5, 521-529.

Linnakoski, R., Puhakka-Tarvainen, H., \& Pappinen, A. (2012). Endophytic fungi isolated from Khaya anthotheca in Ghana. Fungal Ecology, 5, 298-308.

Meriles, J. M., Lamarque, A. L., Labuckas, D. O., \& Maestri, D. M. (2004). Effect of fungal damage by Fusarium spp and Diaporthe/Phomopsis complex on protein quantity and quality of soybean seed. Journal of the Science of Food and Agriculture, 84(12), 1594-1598.

Pioli, R. N., Benavídez, R., \& Morandi, E. N. (1997). Estudio preliminar sobre la incidencia de patógenos en semillas de soja fresca para consumo humano. Fitopatología, 32, 106-120.

Pioli, R. N., Benavídez, R., Morandi, E. N., \& Bodrero, M. (2000). Epidemiological study of diseases associated to soybean carpels and seeds, Santa Fe, Argentina. Fitopatología, 35(2), 111-118.

Pioli, R. N., Morandi, E. N., \& Bisaro, V. (2001). First report about Soybean stem canker caused by $D$. phaseolorum var. caulivora. Plant Disease Note, 85(1), 95.

Pioli, R., Morandi, E. N., \& Luque, A. (2002). Outbreak of Soybean stem canker caused by D. phaseolorum var. caulivora in Argentina. Plant Dissease Note, 86(12), 1403.

Pioli, R. N., Morandi, E. N., Martínez, M. C., Lucca, M. F., Tozzini, A., Bisaro, V., \& Hopp, H. E. (2003). Morphological, molecular and pathogenic characterization of Diaporthe phaseolorum variability in the soybean producing area of Argentina. Phytopathology, 93(2), 136-146.

Pioli, R.N., \& Morandi, E. 2005. Diaporthe phaseolorum var. sojae. In Crop Protection Compendium. Texto, imágenes, distrib. mundial y otros. $\mathrm{CAB}$ Internacional. Published in CD ROM and Internet vertions. Wallingford, UK: L. Mcgillivray.

Ponce de León, C., Torija, M. E., \& Matallana, M. C. (2013). Utilidad en la alimentación de algunas semillas germinadas de soja y trigo. Boletín de la Real Sociedad Española de Historia Natural Sección Biología, 107, 47-55.

Promputtha, I., Jeewon, R., Lumyong, S., McKenzie, E. H. C., \& Hyde, K. D. (2005). Ribosomal DNA fingerprinting in the identification of non sporulating endophytes from Magnolia liliiflora (Magnoliaceae). Fungal Diversity, 20, 167-186. 
Rossman, A. Y., Farr, D. F., \& Castlebury, L. A. (2007). A review of the phylogeny and biology of the Diaporthales. Mycoscience, 48, 135-144.

Saikkonen, K. (2007). Forest structure and fungal endophytes. Fungal Biology Reviews, 21, 67-74.

Sakadilis, M. L., Hardy, G. E. S. J., \& Burgess, T. I. (2011). Endophytes as potential pathogens of the baobab species Adansonia gregorii:a focus on the Botryosphaeriaceae. Fungal Ecology, 4, 1-14.

Santos, J. M., \& Phillips, A. J. L. (2009). Resolving the complex of Diaporthe (Phomopsis) species occurring on Foeniculum vulgare in Portugal. Fungal Diversity, 34, 111-125.

Santos, J. M., Vrandecic, K., Cosic, J., Duvnjak, T., \& Phillips, A. J. L. (2011). Resolving the Diaporthe species occurring on soybean in Croatia. Persoonia, 27, 9-19.

Udayanga, D., Liu, X. X., McKenzie, E. H. C., Chukeatirote, E., Bahkali, A. H., \& Hyde, K. D. (2011). Genus Phomopsis: biology, applications, specie concepts and names of common phytopathogens. Fungal Diversity, 50, 189-225.

Vidić, M., Jasnic, S., \& Miladinovic, J. (1995). Pathogenicity of P. sojae and P. longicolla. Zastita-Bilja, 46(3), 197-205.

Vidić, M., Jasnić, S., \& Petrović, K. (2011). Vrste roda Diaporthe / Phomopsis na soji u Srbiji. Pestic Fitomed (Beograd), 26(4), 301-315.

Ying-Hui, L., Wei, L., Chen, Z., Liang, Y., Ru-Zhen, C., Brandon, G., \& Li-Juan, Q. (2010). Genetic diversity in domesticated soybean (Glycine max) and its wild progenitor ( $G$. soja) for sequence repeat and singlenucleotide polymorphism loci. New Phytologist, 188, 242-253.

Zhang, A., Riccioni, L., Pedersen, W., Kollipara, K., \& Hartman, G. (1998). Molecular identification and phylogenetic grouping of $D$. phaseolorum and $P$. longicolla isolates from soybean. Phytopathology, $88,1306-1314$. 